Mots. Les langages du politique

\title{
Bling-bling. Du hip-hop aux dictionnaires, en passant par les médias
}

\section{Carmen Alén Garabato}

\section{(2) OpenEdition \\ Journals}

Édition électronique

URL : https://journals.openedition.org/mots/21172

DOI : $10.4000 /$ mots. 21172

ISSN : 1960-6001

Éditeur

ENS Éditions

\section{Édition imprimée}

Date de publication : 22 avril 2013

Pagination : 81-96

ISBN : 978-2-84788-393-0

ISSN : 0243-6450

\section{Référence électronique}

Carmen Alén Garabato, «Bling-bling. Du hip-hop aux dictionnaires, en passant par les médias », Mots. Les langages du politique [En ligne], 101 | 2013, mis en ligne le 22 avril 2015, consulté le 22 avril 2022. URL : http://journals.openedition.org/mots/21172 ; DOI : https://doi.org/10.4000/mots.21172 


\section{Bling-bling. Du hip-hop aux dictionnaires, en passant par les médias}

Bling-bling fait partie de l'ensemble des mots qui sont entrés dans l'édition de 2010 du dictionnaire Le Petit Robert. Cet emprunt à l'anglo-américain peut fonctionner, d'après ce dictionnaire, comme nom masculin invariable ou comme adjectif : «Qui affiche un luxe ostentatoire et clinquant». Un aperçu de son utilisation dans la presse française met en évidence que cette définition ne rend pas compte de la «teneur populaire du mot, c'est-à-dire des échos implicites qu’il éveille et véhicule dans la communauté linguistique» (Pruvost, 2005, p.17). Je vais m'intéresser ici à la trajectoire de cet emprunt dans la presse française et à la «charge culturelle partagée» (Galisson, 1988) que ce terme a acquise au fil des années en France (notamment dans le débat politique, mais pas seulement), sans par ailleurs perdre sa signification première.

\section{Les origines du mot et ses débuts en France}

D'après l'Oxford English Dictionary', bling est un terme d'argot issu du langage du rap et du hip-hop utilisé fréquemment avec redoublement : blingbling. Il serait probablement une imitation représentant l'effet visuel de la lumière réfléchie par des pierres ou métaux précieux. Il est utilisé en anglais comme substantif (désignant un/des bijou(x) ostentatoire(s), et, en conséquence, l'étalage de la richesse, de la consommation) ou comme adjectif qui signifie «tape-à-l'œil» (pour un bijou flamboyant ou une robe), ou qui glorifie la consommation ostentatoire.

Ce néologisme arrive en France dans les années deux mille, ce qui a été le cas de beaucoup d'autres termes appartenant au domaine de la musique (rap, hip-hop, slam). Du point de vue formel, il s'agit d'un cas de non-intégration d'un mot étranger, qu'elle soit phonétique (sa prononciation n'est pas conforme aux normes du français) ou graphique ; mais ce mot a tout de même été intégré au

1. Oxford English Dictionary, troisième édition, juin 2006 ; version en ligne, juin 2011. 
système lexico-grammatical du français, si l'on tient compte du fait qu'il peut recevoir les marques de pluriel (bling-blings, blings-bling) ainsi que certaines dérivations suffixales : bling-blinguiser, bling-blingant, bling-blingissime, bling-blinguerie, bling-blinguisation... (formes trouvées dans le corpus de presse dont il sera question plus loin)2.

Du point de vue sémantique, l'adoption de cet emprunt produit une « double réorganisation sémantique» (Charaudeau, 1992, p. 83) du lexique français:

- d'un côté, bling-bling "se présente accompagné d'une réalité étrangère qui n'a pas d'existence dans la culture de la langue d'accueil» (un style de musique, un type de pendentif...), et il enrichit le lexique de celle-ci par « accumulation» (ibid., 1992, p. 83);

- d'un autre côté, «le mot emprunté entre en concurrence avec des mots et des réalités proches dans la langue d'accueil. Le lexique de celle-ci s'enrichit en étendant son champ de synonymes » (ibid.), en l'occurrence : «tape-àl'œil», «clinquant», «parvenu»...

Les procédés de réorganisation sémantique du lexique de la langue d'accueil sont soumis, comme le signale encore Patrick Charaudeau, «à des influences d'ordre social, économique et culturel ». Dans le cas de bling-bling, «la force des mouvements de mode » et notamment du «langage des médias» a été déterminante dans sa promotion : elle a favorisé son intégration et l'a fait perdurer dans le temps au point d'aboutir à une assimilation que l'on pourrait considérer comme accomplie. Cet anglicisme est devenu en peu de temps un mot très fréquemment utilisé dans les médias français, d'où, on le verra, il a été exporté vers d'autres langues romanes avec les connotations socio-politiques qu'il a acquises en France. Mais bling-bling n'a pas perdu son premier emploi : il «apparait toujours en filigrane et influence les emplois ultérieurs» (Sablayrolles, 1993, p. 223).

\section{L’itinéraire de bling-bling dans la presse française}

Selon le répertoire d'Europresse ${ }^{3}$, bling-bling 4 apparaît pour la première fois dans la presse en France le 9 mai 1997 : on le trouve dans Sud Ouest, non pas comme un emprunt à l'anglo-américain mais comme une onomatopée imitant

2. D'après Le Petit Robert, il s'agit pourtant d'un mot invariable.

3. Nous avons interrogé la base de données Europresse pour tracer l'itinéraire de ce mot en France, et notamment son utilisation dans la presse généraliste (nationale et régionale). Les citations qui se trouvent dans cet article ont été extraites des documents répertoriés dans cette base, à l'exception de celles pour lesquelles nous indiquons une autre source. La période prise en compte commence le 9 mai 1997 (date de la première apparition du mot en France) et se termine le 30 septembre 2011, date à la quelle la rédaction de cet article s'est terminée. La base de données Factiva a été utilisée pour avoir accès à la presse étrangère.

4. On trouve le mot écrit de façons différentes : «Bling-Bling », «bling bling », «bling, bling »... 
le son de certains instruments musicaux 5 : "[...] chaque musicien bruitait son instrument (ploum-ploum, tchac-tchac, bling-bling, etc.) [...]».

En tant qu'emprunt, le fonctionnement sémantico-référentiel de bling-bling évolue rapidement à partir des années deux mille :

1. Durant les premières années de la décennie, le fonctionnement onomatopéique se maintient mais dans l'univers de référence anglo-saxon. On emprunte donc «la dénomination en même temps qu'on importe une nouvelle réalité» (Pruvost, Sablayrolles, 2003) : l'expression est utilisée à plusieurs reprises dans Libération au sein d'articles consacrés à la musique et désignant les bijoux (et le bruit qu'ils produisent) portés par certains membres de groupes de hip-hop :

[...] le retour du hip-hop... - en tout cas d'un hip-hop prenant le contre-pied d'un rap surcommercialisé, faisant l'éloge des marques de haute couture (Gucci, Versace...) et des chaînes en or qui font «bling, bling». (Libération, 4 décembre 2000)

Sur une musique synthétique, les Hot Boyz glorifient leurs bijoux (Bling, Bling) [...]. (Libération, 3 février 2001)

Par extension, il désigne un groupe ou un style de musique ${ }^{6}$ :

[...] les 100 Meilleurs Clips de Bling Bling. (Libération, 6 septembre 2001)

2. L'expression élargit par la suite son fonctionnement sémantico-référentiel et est associée à une esthétique qui semble prendre de l'ampleur, décrite à partir d'accessoires voyants : «bijoux-breloques-gris-gris», «sacs perlés» et «boas» (Le Progrès, 5 septembre 2004), «le culte des Lexus et des chaînes en or» (Le Monde, 30 juin 2005), «bijoux clinquants, voitures flamboyantes, vêtements de designers, une avalanche de diamants en strass» (Le Figaro, 28 février 2006), « grosses cylindrées, bateaux et filles en bikini... » (Sud Ouest, 13 janvier 2008)... Le fonctionnement onomatopéique ne disparaît pas, il ajoute une certaine dérision à l'esthétique décrite.

3. L'utilisation du terme dépasse rapidement le domaine musical : il est récupéré par le marketing et on le trouve dans les rubriques de mode; ainsi, dès le 5 septembre 2004, celle du Progrès parle de la «tendance Bling-Bling». Par ailleurs, dans Le Figaro, on commente une exposition de broderie organisée à Zurich, qui prend comme titre Bling-bling pour montrer des «tissus de rêve de Saint-Gall au Musée national suisse» (Le Figaro, 18 novembre 2004). Cette «tendance ostentatoire n'échappe pas au high-tech », comme le rappelle Le Point du 4 juillet 2006 lorsqu'il présente les «Produits du futur bling-bling »: « téléphone portable, [...] en or jaune ou blanc 18 carats, incrusté de diamants

5. Le mot apparaît, avec une signification similaire, dans la forme simple bling dans d'autres journaux (3 occurrences en 4 ans).

6. Premières apparitions dans la presse de langue anglaise en 2000 : dans The New York Times et The Canadian Press. 
et inspiré de pièces de joaillerie »; « un écran de 71 pouces et doré à l'or fin »; «interrupteurs dorés et argentés»; téléphones «dans une robe dorée insolente »... Le Figaro parle de la «bling-bling attitude», qui

est de mise chez les traiteurs, dans les épiceries fines et les grands restaurants. [...] L'Europe découvre les vertus décoratives de l'or. Apparues sur les ganaches des chocolatiers, les paillettes du métal jaune illuminent désormais les macarons (notamment chez Pierre Hermé), les éclairs, les calissons, le thé, les vodkas, les huiles, voire les confitures. (Le Figaro, 30 décembre 2006)

Bling-bling devient un mot qui attire l'attention des journalistes... et des lecteurs. On trouve de nombreuses rubriques qui lui sont consacrées et qui retracent son parcours en France. Ainsi Le Point insiste sur sa rentabilité et lui consacre un bref article le 21 décembre 2006 (Marketing) en l'intitulant : "Le "bling bling", un filon en or »:

Un certain temps s'est écoulé entre l'apparition du terme «bling bling », qui désigne l'attirail ostentatoire des rappeurs américains, et sa récupération par les pros du marketing. Mais, depuis quelques semaines, le clinquant dégouline des rayons. La marque de cosmétiques Hard Candy vient de lancer le fard pailleté Bling Bling pour les yeux, Boucheron propose une eau de parfum Diamant à 72 euros les 50 millilitres. Au rayon boissons, la vodka Bling Bling d'Absolut a le même goût qu'une vodka classique, mais sa bouteille recouverte d'or permet à la marque suédoise de la vendre plus de 12 euros le litre. Dans le genre «poudre aux yeux», on trouve l'eau Bling $\mathrm{H}_{2} \mathrm{O}$, dont la bouteille est cloutée de cristaux Svarovski. Et aux États-Unis, les garagistes proposent des jantes en or massif dont le prix peut atteindre $50000 \mathrm{dol}$ lars pièce. Avec cette nouvelle tendance, c'est surtout la caisse enregistreuse qui fait «bling bling ». (Mélanie Delattre)

Le mot bling-bling devient ainsi un élément clé de la recherche de spectacle propre au discours journalistique (Lochard, Boyer, 1998, p. 24). Cela explique sa présence très fréquente dans les titres (ou les sous-titres), comme le montre le graphique ci-dessous qui indique le nombre de textes dont le titre (ou le sous-titre) contient le mot bling-bling entre 2006 et 2010 dans notre corpus :

Ces titres ont comme objectif d'attirer le lecteur et de situer le texte qui suit dans un contexte socio-politique ou socio-culturel. Le mot bling-bling se prête sans beaucoup d'effort au spectacle (voir ci-dessus les fonctions référentielle et expressive du terme, soulignées par certains périodiques). Pour reprendre les termes de Lochard et Boyer - «le "choc" des mots ou des images participe bien du principe de plaisir auquel est assujetti tout discours médiatique»-, on montre les bijoux flamboyants des rappeurs, un restaurant de Dolce \& Gabbana à Milan, Gold, où tout est doré... Mais on fait du spectacle également sur une exposition, sur la visite de la chancelière allemande... : les événements les plus variés deviennent des occasions de faire jouer le «mot vedette» (Boyer, 2003, p.118): 


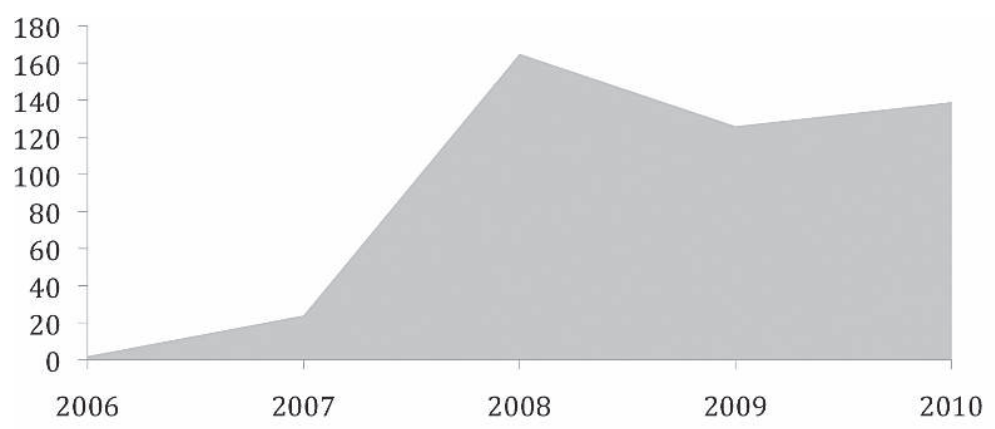

Graphique 1

- Gaza à l'heure du bling-bling (Marianne, 16 juillet 2011)

- De la droite bling-bling à la gauche parvenue (Marianne, 18 juillet 2011)

- Pour une recherche bling-bling? (Le Monde, 19 février 2009)

- Les «Ch'tis», l'anti-Sarkoland, l'anti-bling-bling (Libération, 31 décembre 2008)

- Face au président bling-bling, une maire brique-brique (La Voix du Nord, 24 janvier 2010)

- Le mariage «bling-bling » se termine à la fourrière (Le Bien public, 7 mai 2010)

- L’UMP dénonce des vœux bling-bling (Le Parisien, 6 janvier 2011)

- Le cœur de Paris redessiné sur le mode bling-bling (Le Parisien, 2 juillet 2011) Ces quelques exemples, qui ne rendent pas totalement compte de l'abondante utilisation du terme, montrent l'élargissement des domaines dans lesquels l'expression est rapidement utilisée en France7. Au fil des années, bling-bling comme adjectif peut être appliqué aux substantifs les plus variés, désignant :

- des référents appartenant au monde de la musique (dont il procède) : artistes, chanteurs, clichés, clips, dj, hip-hop, rap, rappeur, rumba, tube...;

- des accessoires du monde de la mode : colliers, colifichets, fard pailleté, lunettes de ski, bijouterie, imagerie, lèvres, montre, bracelets...;

- des objets divers : bouteille, horloge, interrupteur, machine, voiture...;

- des boissons : apéro, cognac, vodka, cocktails...;

- de la nourriture : poulet, poisson, pizza...;

- des villes, pays ou continents : Paris, Italie, France, Russie, Amérique, Chine...;

- des personnages publics : Nicolas Sarkozy, Carla Bruni, Chirac...;

- des sportifs : boxeur, footballeur...

7. Dans plus de 6000 documents entre 1997 et 2011 d'après la base Europresse. 
Mais bling-bling peut aussi qualifier une époque (années, génération...), une tendance ou un style (esthétique, ambiance, atmosphère, architecture, art, cinéma, culture, littérature...), la charité, les vacances, la cuisine, etc.

4. Mais ce mot devient aussi peu à peu un motstigmate (Boyer, 1990), voire un mot-tabou ${ }^{8}$ (Reboul, 1980, p.113) : une nouvelle connotation péjorative (mais non généralisée) apparaît lorsqu'on parle par exemple des «clips “bling bling”, gorgés de dollars, de champagne, de villas immaculées, de machos moches et de bombes en string» (Le Parisien, 31 juillet 2006). On définit le "bling-bling » comme étant « la course à la frime et à l'argent qui noyaute le hiphop au détriment de la dimension artistique » (L'Humanité, 21 août 2006), ou on le caractérise par « des bagues surdimensionnées, des colliers plastron en or massif, ou des bijoux cylindriques empruntant au vocabulaire automobile " (Le Figaro, 2 novembre 2006). Cette charge référentielle sera reprise et intensifiée lors de l'application du terme au domaine de la politique.

C'est bien là l'originalité française : le terme bling-bling va être associé au style, jugé ostentatoire, de Nicolas Sarkozy et de son entourage politique. Dans ce contexte, bling-bling devient le mot clé d'un discours conflictuel (Windisch, 1987) qui oppose la presse française au président de la République (et à la droite au pouvoir). Dans ce «K-O. verbal» est disqualifié «non seulement un discours, mais une personne et son identité» (ibid., p. 27). Comme le dit Windisch, «dans le cas d'une communication conflictuelle, il ne s'agit plus seulement de transmettre sagement une information [...]. D'autres fonctions sociales de la langue peuvent compléter sa fonction première de communication» (p.18). Dans le cas qui nous occupe, il s'agit bien de disqualifier, de dénoncer, de ridiculiser...; bling-bling sera chargé d'« effets discursifs durables» liés à «la prise de parti dans un débat social» : on assiste à une "déformation» (Laurendeau, 1997, p. 27-29) consciente et volontaire du terme.

Le premier article à faire une association dans ce sens est publié dans Le Point le 3 mai 2007, durant la campagne présidentielle :

De A à Z, les attaques contre Sarkozy et Royal

Les attaques contre Sarkozy [...]

\section{Bing-bling}

À part Doc Gyneco, les rappeurs n'aiment pas Sarkozy. Pourtant, ils ne manquent pas de remarquer qu'il porte (presque) la même panoplie. Lunettes Ray-Ban, chaîne en or, chronographe Breitling au poignet : comme eux, le candidat de l'UMP aime les marques, le luxe et ce qui brille. Bref, ce que, dans la mythologie hip-hop, on appelle le «bling-bling».

8. Olivier Reboul distingue les mots-chocs («ces termes ou ces expressions qui ont par eux-mêmes, du fait de leur forte connotation, un résultat incitatif, quelle que soit la phrase dans laquelle ils s'insèrent») des mots-tabous ("les mots-chocs qui produisent d'eux-mêmes un effet négatif, qui disqualifient ce qu'ils désignent») (Reboul, 1980, p.113). 
Après l'élection, deux semaines plus tard, Libération parle de «la blingbling intronisation de Sarkozy mercredi à l’Élysée » (Libération, 19 mai 2007), le jour même où Nicolas Domenach publie dans Marianne un article (dans un discours ironique-conflictuel - voir Windisch, p.117), intitulé «La droite "blingbling" $»$ :

Quand ils se moquent d'eux-mêmes, à force de faire sonner leurs portables argentés et cliqueter leurs bijoux en or, les rappeurs s'appellent les «bling-bling». Désormais, il faut compter aussi avec «la droite bling-bling». La droite sarkozyste a célébré sa revanche sur la gauche caviar et le gaullisme mormon en prenant d'assaut le Fouquet's. Pourquoi payer si cher pour mal manger? Pour que ça se voie!

Et, qu'on se le dise, nous avons « un nouveau président sans tabou» qui ose l'impossible : s'afficher pour quelques jours de vacances en famille sur le yacht, avec jacuzzi s'il vous plaît, d'un ami milliardaire, fier d'aider à régénérer la force de travail d'un homme politique qui se lève tôt. Audace et modernité inouïes, immédiatement saluées par cette France qui se couche tôt parce qu'elle rentre de boîte de nuit et bientôt de Suisse, puisqu'on va lui réduire ses impôts. La droite bling-bling est celle du ressentiment fiscal. La droite de la bulle de champagne.

[...]. On ne dira jamais assez l'importance de la lutte des classes à Neuilly. L'humilié, le petit Nicolas, a pris sa revanche. Sur le yacht de son «pote», comme un bras d'honneur avec Rolex au poignet. Bing-bling... [...]

Ces deux textes contribuent à la « construction du sens » : l' "énonciateur» et l'« interprétant» partagent désormais le même filtre et la nouvelle signification de bling-bling constitue un "savoir partagé » (Charaudeau, 1983, p. 24).

L'emballement médiatique que subira ce mot se déclenche en décembre 2007 (et coïncide avec la publication des clichés qui montrent Nicolas Sarkozy et Carla Bruni à Disneyland Paris). Libération publie alors la photo de Sarkozy avec le titre Président bling-bling (voir document en annexe). Les occurrences du terme se multiplient les mois suivants dans pratiquement toute la presse française, aussi bien nationale que régionale. Il s'insère, lorsqu'il est question de politique, dans un discours fortement conflictuel (Windisch, 1987) dont bling-bling est le mot clé. Les nombreux dérivés du nom du président que l'on peut trouver dans les documents qui utilisent le terme bling-bling font état de cette fixation des médias sur le personnage présidentiel : sarko-addiction, sarkoberlusconisme, sarckoboy, sarko-circus, sarkocompatible, sarkoconvertis, sarkocratie, sarko-doléance, sarko-France, sarkohéros, sarkoïser, sarkolalie, sarkoland, sarkolâtrie, sarkoléon, sarkoleptique, sarkologue, sarkomanie, sarkonoïa, sarkonomics, sarkophage, sarkophilie, sarkophobie, sarkophrénie, sarko-populisme, sarkopragmatisme, sarkosarkozyste, sarkose, sarko-show, sarko-telling, sarkoverdose, sarkowii, sarkozade, sarkozette, sarkozie, sarkozien, sarkozix, sarkoso-dépendant, sarkozie, sarkozysation, sarkozisto-bling-bling... Le Point, Marianne et Libération se disputent la paternité de la mise en discours politique du terme : «Bling-bling c'est nous!», 
titre Le Point le 3 janvier, rappelant que le mot avait été utilisé dans l'hebdomadaire le 3 mai 2007. Ce à quoi répond Marianne :

Le Point se la pète

Voilà que l'hebdomadaire Le Point exhume de ses caves un article passé totalement inaperçu afin de toucher des royalties de reconnaissance sur l'expression «droite bling-bling» dont nous revendiquions, preuve à l'appui, la paternité. L'organe directeur du sarkozysme pensant avait bien écrit entre deux pubs que «le candidat de l'UMP aime [...] ce qui brille. Ce que, dans la mythologie hip-hop, on appelle le "bling-bling" », mais le faraud n'avait pas élaboré le concept de «droite bling-bling » que Marianne a forgé. (Marianne, 12 janvier 2008)

\section{" La coqueluche des médias "9 français...}

Le terme bling-bling continue sans faiblir à être associé à ce style présidentiel, mais ne s'y circonscrit pas, contrairement à ce qu'on pourrait penser. Sa médiatisation associée au président semble favoriser son utilisation dans beaucoup d'autres domaines. Le graphique 2 ci-après montre le rapport entre l'augmentation du nombre d'articles dans lesquels apparaît le terme bling-bling et son association à Nicolas Sarkozy et à la droite politique ${ }^{10}$. Mais il met aussi en évidence que la fortune du mot ne leur est pas exclusivement liée. Comme il a été observé plus haut, il peut être aussi question de musique, de mode, de boissons, de nourriture, de sportifs, etc.

Dans cette dernière partie, je mettrai en évidence le rôle des médias français dans la diffusion de cet emprunt à l'anglais. On sait que beaucoup de néologismes «naissent» et «prennent leur élan» dans la presse écrite (et audiovisuelle), qui devient ainsi «un lieu privilégié du débat» autour de leur légitimité (Pruvost, Sablayrolles, 2003, p.16). En ce qui concerne bling-bling, il s'agit bien d'une «focalisation lexico-pragmatique » qui «n'est pas innocente», autrement dit d' "une pratique qui consiste à promouvoir massivement l'usage d'un mot (ou éventuellement de plusieurs) : mot "totem", "slogan" ou simplement "vedette", durant une période plus ou moins brève» (Boyer, 2003, p.118). Ainsi, dans un premier moment, la presse use d'un procédé métalinguistique : un commentaire explicatif fait par l'énonciateur à propos d'un mot dont le sens «ne va pas de soi » (Authier-Revuz, 1994, p. 91). Lorsque le terme entre dans le «langage commun », les efforts se centrent sur sa consécration dans les dictionnaires.

9. Expression employée dans un article de Bruno Lawaele paru dans la Voix du Nord le 20 janvier 2008.

10. Il s'agit des documents dans lesquels on trouve le terme «bling-bling » en même temps que «Sarkozy» ou «président» ou «présidence» ou «droite». 


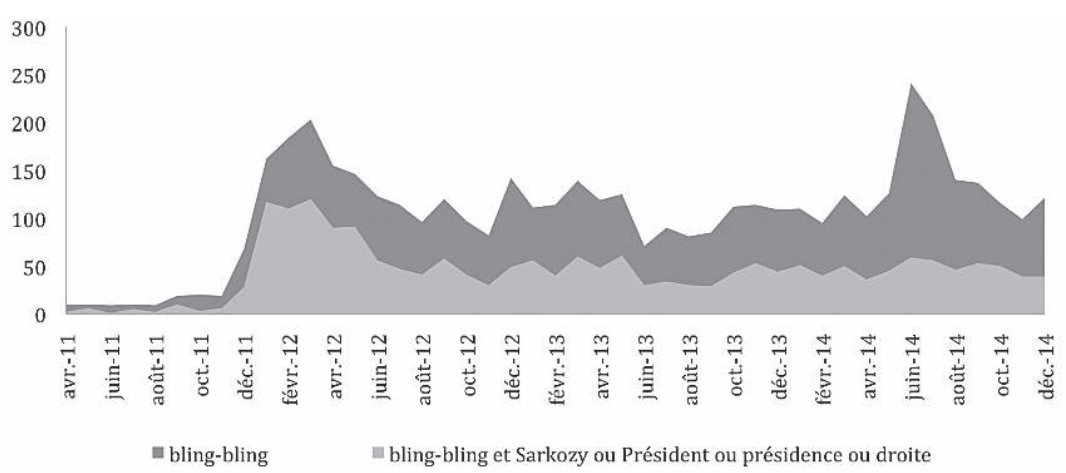

Graphique 2

S'agissant d'abord d'un xénisme, lors des premières utilisations du mot, les gloses ${ }^{11}$ sont fréquentes. Elles peuvent prendre des formes différentes. Ce sont parfois de simples traductions présentées entre parenthèses :

- bling bling (chaînes en or) (Libération, 28 octobre 2005)

- «bling-bling» (bijoux clinquants, voitures flamboyantes, vêtements de designers) (Le Figaro, 4 novembre 2005)

- «bling bling» (traduction : chaînes en or et autres bijoux qui brillent) (Le Progrès, 28 janvier 2006)

- «bling-bling» (une bijouterie voyante...) (Le Point, 6 avril 2006)

Parfois, ce sont des traductions présentées comme «notes de la rédaction », qui explicitent la signification du mot dans son contexte :

- bling bling (bijoux, ndlr) (Libération, 17 février 2005)

- bling-bling (friqué, ndlr) (Libération, 16 août 2005)

- les bling-bling (colliers ostentatoires qu'affectionnent les rappeurs, ndlr) (Libération, 18 avril 2006)

Parfois encore, des commentaires métalinguistiques plus élaborés, qui peuvent prendre plusieurs lignes et apporter des éclaircissements sur le sens (propre ou figuré) mais aussi sur l'origine du mot concerné :

- Bling Bling : une onomatopée reproduisant le bruit d'ouverture d'un tiroir-caisse. (Le Monde, 20 octobre 2001).

- Le «bling bling» est un ensemble formé d'une grosse chaîne ornée d'un énorme pendentif. De nombreux rappeurs américains en portent plusieurs qui s'entrechoquent, d'où leur nom. (Le Télégramme, 28 septembre 2006)

11. Voir, à propos de ces brefs commentaires métalinguistiques, Steuckardt, Honoré, 2006. 
- Bling-bling : se dit des artistes adoptant un style clinquant, mettant en avant leur argent. (Le Parisien, 25 novembre 2006)

- [...] la mode «bling bling». C'est un terme issu du jargon hip-hop américain qui désigne le style ostentatoire et excessif des rappeurs milliardaires d'outre-Atlantique, surtout ceux de la côte Est. (Le Parisien, 21 avril 2007)

- [...] rap bling-bling (nom donné aux rappeurs populaires qui portent des grosses chaînes et bijoux). (Ouest-France Bretagne, 3 août 2007)

- [...] la culture «bling-bling» (l'onomatopée désigne le tintement de gros bijoux), un terme issu du rap qui désigne la façon d'étaler sans complexe, voire avec ostentation, des signes extérieurs de réussite sociale. (Le Monde, 25 août 2007)

- «Bling-bling», l'expression vient des rues d'outre-Atlantique où des rappeurs affichent un luxe d'objets tape-à-l'oeil. (La Croix, 19 décembre 2007)

À partir de 2007 , ce type de gloses disparaît pratiquement : ce serait une preuve que le mot est entré dans l'usage et que sa signification est bien connue du lecteur. Mais à leur place apparaissent des articles, plus ou moins longs, ayant pour objet principal de tracer les origines et l'histoire de bling-bling en France. Les titres de ces articles cherchent à attirer l'attention sur ce nouveau mot (c'est moi qui souligne) :

- LEMOTÀ RETENIR. Bling bling (Aujourd'hui en France, 28 juillet 2007)

- Regards. Un mot. Bling-bling (Ouest-France, 27 décembre 2007)

- Le mot du jour. Bling-bling. (Aujourd'hui en France / Le Parisien, 30 décembre 2007)

- Langage. Du hip-hop à la jet-set : on se lève tous pour faire bling-bling! (La Voix du Nord, 20 janvier 2008)

- Une expression dans l'air du temps (Le Monde, 18 février 2008)

- Actualité. Vocabulaire. Ça fait bling-bling (Sud Ouest, 13 janvier 2008)

Des synonymes sont parfois proposés :

- Un synonyme, en quelque sorte, de nouveau riche. (Le Parisien, 28 juillet 2007)

- Synonyme : "tape à l'œil», "Attention, c'est moi que v'la». (Aujourd'hui en France / Le Parisien, 30 décembre 2007)

Mais donner des synonymes ne signifie pas critiquer ou mettre en question le besoin de ce nouveau terme emprunté à l'anglais, carbling-bling acquiert en France « une valeur d'identité sociale qui justifie son maintien » (Charaudeau, 1992, p. 105). Ainsi, il est question d'une "expression dans l'air du temps», de la "génération bling-bling», de la «culture bling-bling», de la «période bling-bling», des «années bling-bling», de l'«époque bling-bling »... Représentant un moment concret de l'histoire de la France, la valeur référentielle du mot empêche son remplacement par un synonyme appartenant à un « langage d'autrefois » dont il prendrait ainsi le relais :

Bref, les bling-bling d'aujourd'hui seraient en quelque sorte les «parvenus » d'autrefois. (Ouest-France, 27 décembre 2007) 
Et lorsque Libération (31 janvier 2009) se demande pourquoi Ségolène Royal a utilisé l'expression m'as-tu-vu, «vieillotte, un peu balzacienne en un sens», au lieu de bling-bling, "passé dans la langue commune» (pour parler du président de la République), la réponse est : «Ségolène Royal a écrit “m”astu-vu" parce que, comme elle l'explique elle "vient du xıxe siècle".»

Certains journaux soulignent par ailleurs la «fonction d'expressivité» résultant «de l'usage qui est fait» (Charaudeau, 1992, p.65) de ce nouveau mot :

Les parvenus d'aujourd'hui sont plus sympathiquement bling-bling. (Le Monde, 8 janvier 2008)

Bling-bling. Ça brille, ça claque, ça marque. (Libération, 31 décembre 2009)

«Bling-bling». Ah, le fameux... Sonore, scintillant, limite toc. (Le Parisien, 11 juin 2008)

Mais la consécration de ce terme promu par les médias sera son enregistrement dans Le Robert. Le rôle de la presse est à nouveau important : Alain Rey est vivement critiqué lorsqu'il décide de ne pas l'inscrire dans l'édition de 2008. Plusieurs journaux plaident en sa faveur. C'est le cas de l'AFP Infos françaises, qui retrace le 12 janvier 2008 l'histoire du mot (je souligne) :

Pour l'instant, les principaux dictionnaires français ne l'ont pas encore accueilli dans leurs pages. Mais certains lexicographes, chargés de repérer les nouveaux mots et de rédiger les dictionnaires, le pistent avec délice. [...] les bling-bling sont légion, ce qui agace Alain Rey, conseiller éditorial pour Le Robert. «Le mot français clinquant est aussi parlant », déclare-t-il à l'AFP [...]. Alors, à quand bling-bling dans un dictionnaire français? « Quand la discussion viendra surce mot au Robert, je serai assez réticent. Cela risque de ne pas durer», considère Alain Rey. Chez Larousse aussi, on ne veut pas se précipiter sur un mot qui pourrait ne pas être pérenne.

La Voix du Nord (20 janvier 2008) plaide dans le même sens quelques jours plus tard (je souligne) :

Dur, dur... pour les dicos! Le moins drôle de l'affaire n'est pas que nos lexicographes, encore une fois, se font ostensiblement tirer l'oreille. Renseignements pris, ni Larousse ni Robert ne seraient enthousiastes à l'idée d'accueillir, dans un magasin de porcelaine qui compte déjà toc et tape-à-l'œil, cet éléphant-là. Passe pour le premier nommé, qui, en la matière, ne s'est jamais départi d'une insigne prudence. Mais voilà qui est moins habituel de la part du second, lequel s'est toujours targué, pour citer Jacques-Pierre Amette, de « laisser passer tous les courants d'air de notre époque». Toujours rué - à donf, quoi - à la rencontre du néologisme le plus kitsch. Entendre Alain Rey faire la fine bouche devant ce vocable inutile, sous prétexte que "le mot français clinquant est aussi parlant», en déconcertera plus d'un : s'il fallait bannir de nos dictionnaires tous les anglicismes superfétatoires, on réaliserait de fameuses économies de papier! Quant à ses «réticences» à l'égard d'un mot qui « risque de ne pas durer», gageons qu'elles arracheront un sourire aux familiers de son Petit Robert, depuis longtemps jeté en pâture aux zarb, keuf, ouf et autres fleurs plus ou moins périssables des banlieues. Allez, tchao-tchao! 
L'absence annoncée du terme dans l'édition de 2008 du Dictionnaire Le Robert est confirmée en juin. La Nouvelle République du Centre-Ouest la regrette le 12 juin (je souligne) :

Dommage pour «bling bling», recalé lui aussi, même si cette onomatopée qui a fait «bing» est employée maintenant par plus de 60 millions de Français. «"Bling bling” est trop attaché à Nicolas Sarkozy », explique Alain Rey, le pape de la langue française et gardien du temple des bonnes manières linguistiques. Il devrait savoir pourtant que le chef de l’État a laissé sa Rolex au vestiaire.

Et Marianne publie deux jours plus tard (le 14 juin 2008) un bref article sur un ton d'indignation intitulé : «Dico. Complètement bling-bling ». Il est question dans son plaidoyer de la fonction référentielle du mot (Charaudeau, 1992, p.65), de sa charge politique et polémique (je souligne) :

Alain Rey est aveugle idéologiquement. Tentons de lui dessiller les yeux. Quand nous avons forgé le concept de droite et de président "bling-bling», nous ne visions pas seulement un exhibitionnisme tapageur, mais nous cernions, nous stigmatisions une attitude politique inégalitaire. Ce «bling-bling» qui résume et symbolise le Fouquet's comme la loi Tepa est plus qu'un comportement : c'est un manifeste ostentatoire, une stratégie, qui privilégie les riches et l'illusion que leur enrichissement ruissellerait jusqu'à ceux d'en bas. Au-delà de l'assurance rappeuse, c'est cette dimension politique qui a fait la fortune du terme, au point qu'il a carillonné dans toutes les têtes, sauf dans celle du « pape de la langue française ». N. D.

\section{Le contre-exemple : bling-bling dans les presses d'autres langues romanes}

J'ai voulu vérifier si la promotion néologique de l'anglicisme bling-bling était réservée à la France ou si elle s'était produite également dans d'autres pays, notamment si d'autres langues romanes l'avaient adopté et avec quelle forme et signification. Le choix des seules langues romanes, certes discutable, présente l'avantage de pouvoir observer le phénomène d'un emprunt à l'angloaméricain dans des langues d'une même famille, au sein de cultures proches. La base de données Factiva ${ }^{12}$ m'a permis d'avoir accès à la presse en espagnol (presse espagnole et sud-américaine), ainsi qu'en catalan, en italien et en portugais (presse portugaise et brésilienne). Force est de constater que le succès du mot en France n'a pas d'équivalent dans les langues et territoires cités.

Dans le corpus de presse d'Amérique Latine, il n'est répertorié que dans environ 70 articles avec la forme bling-bling et moins d'une quinzaine avec la forme blin-blin. Il se trouve dans des contextes en rapport avec la musique et avec les tendances de mode; il est plus rarement utilisé lorsqu'on parle de

12. Nous avons interrogé la base de données (dans son inégralité) en septembre 2011. 
Nicolas Sarkozy ou de son entourage familial. En Espagne, il est moins fréquent et apparaît toujours sous la forme bling-bling : on le trouve deux fois dans $A B C$ (le 29 octobre 2009 et le 11 juin 2010) dans des rubriques consacrées à la mode; El Periódico Mediterráneo l'emploie pour définir un style de musique (le 11 juillet 2010) et El País l'utilise dans le contexte politique français (et européen), en l'expliquant et en consacrant même un court article d'opinion aux “Bling-bling”, dans lequel il dénonce les mesures prises pour expulser les Roms de France avec la caution de l’Europe.

\section{Los Bling Bling}

Utilizada en sus inicios por los raperos más enjoyados, Bling Bling es la populary onomatopéyica denominación que recibe el estilo dominante en la actual derecha francesa. Alude a la ostentación, la presunción y la jactancia. En realidad, la política tintineante del Bling Bling se extiende por Europa. Es un populismo trucho, tramposo, practicado por élites que, en el fondo, desprecian al pueblo al que adulan. [...]. Por eso la comisaria de Justicia europea se ha quedado sola, mientras los Bling Bling que hoy gobiernan Europa van colocando gasas negras en todos los espejos para la gran operación que se ensaya con la expulsión de los gitanos [...]. (El País, 18 septembre 2010) ${ }^{13}$

C'est aussi le cas de La Vanguardia, journal dans lequel on peut lire le 21 août 2011, lorsqu'il est question de la politique de Nicolas Sarkozy :

Paradigma de la ostentación bling bling, su asesor de imagen preferido - le presentó a Carla - resume sus valores. "Quien no tiene un Rolex a los cincuenta años, es un fracasado $» .14$

On trouve également le terme (sous la forme bling-bling) dans la presse en catalan, toujours en rapport avec Nicolas Sarkozy (et seulement dans deux documents) :

Una cerimònia austera que va posar fi a l'estil bling-bling - vocable al.lusiu a les cadenes d'or dels rapers i que designa l'ostentació hortera - del president $i$ va marcar l'inici de 12 mesos de glamur. (El Periódico, 2 février 2009) 15

El de Cécilia Ciganer - Albéniz i el publicista Richard Attias ha estat un casament

13. «Utilisée dans ses débuts par les rappeurs les plus couverts de bijoux, Bling Bling est la dénomination populaire et onomatopéique que reçoit le style dominant dans l'actuelle droite française. Il fait allusion à l'ostentation, à la prétention et à la vantardise. En réalité, la politique tintinnabulante du Bling Bling se répand dans l’Europe. Il s'agit d'un populisme rusé, trompeur, pratiqué par des élites qui, dans le fond, dédaignent le peuple qu'elles flattent [...] C'est pourquoi la commissaire européenne à la Justice est restée isolée, tandis que les Bling Bling qui gouvernent aujourd'hui l'Europe placent des voiles noirs sur tous les miroirs en vue de la grande opération que l'on répète avec l'expulsion des gitans. » (Ma traduction, ici et dans les notes qui suivent.)

14. «Paradigme de l'ostentation bling bling, son conseiller en image préféré - il lui a présenté Carla - résume ses valeurs : “Celui qui n’a pas une Rolex à cinquante ans a raté sa vie.” "

15. "Une cérémonie austère qui a mis fin au style bling-bling - mot qui fait allusion aux chaînes d'or des rappeurs et qui désigne l'ostentation vulgaire - du président et a marqué le début de 12 mois de glamour. » 
una mica bling-bling, vocable amb què els francesos designen l'ostentació amb un punt vulgar que tant agrada - o agradava - a Nicolas Sarkozy, exmarit de la núvia. (El Periódico, 25 mars 2008) 16

L'enquête sur la presse italienne ne fait que confirmer ce que l'on observe dans la presse en espagnol : une utilisation bien plus limitée qu'en France (dans une vingtaine de documents, sous la forme bling-bling ou blin-blin) dans des domaines comme la musique ou la mode et plus rarement pour parler de la politique ou de la vie du président Sarkozy et de sa famille. Enfin, d'après la base de données Factiva, le mot est inexistant en portugais (européen ou non), à une exception près (en tant qu'onomatopée).

On voit donc que le terme bling-bling n'a pas suivi le même processus dans ces pays, où il fonctionne comme emprunt à l'anglais (un xénisme) mais également comme un emprunt au français (xénisme également) avec la charge stigmatisante que le mot a acquise en France.

Dans la société française très médiatisée, l'utilisation et la promotion de bling-bling obéit bien aux impératifs de captation (Lochard, Boyer 1998, p. 24) propres à la communication médiatique. Ce mot a des atouts aussi bien en ce qui concerne sa forme que son contenu (évocation de l'éclat, de la lumière...) : il est ainsi un élément de l'écriture journalistique faisant partie de la recherche de spectacle. Mais son succès est également dû à sa forte valeur référentielle (le Fouquet's, la Rolex, l'or...) indiquant (et stigmatisant) la recherche de visibilité d'une certaine classe politique (ainsi que des chanteurs ou des sportifs).

Mot vedette d'un discours polémique, bling-bling devient en France, en définitive, le centre d'une mise en scène ludique de démasquage, de disqualification (ou même de ridiculisation) de l'adversaire (en l'occurrence Nicolas Sarkozy et la droite politique) sur le mode de l'ironie. Le transfert de ce terme du monde du rap et du hip-hop à celui de la politique (et l'emballement médiatique à propos du goût pour le luxe de la droite au pouvoir) est en quelque sorte l'effet boomerang d'une tendance à la peopolisation ${ }^{17}$ de la politique (exhibition de la vie privée, étalage des richesses). On sait par ailleurs que Nicolas Sarkozy, à la suite de la baisse de sa popularité observée dans les sondages d'opinion, a essayé de corriger son image et s'est affiché dans des cadres plus sobres ${ }^{18}$.

16. «Celui de Cécilia Ciganer-Albéniz avec le publiciste Richard Attias a été un mariage un brin blingbling, mot avec lequel les Français désignent l'ostentation légèrement vulgaire qui plaît tellement - ou qui plaisait - à Nikolas Sarkozy, ex-mari de la mariée.»

17. Voir à propos de la peopolisation de la vie politique Dakhlia, Lherault, 2008.

18. Lors de l'élection présidentielle de mai 2012, le mot bling-bling a été fréquemment utilisé. Le lendemain de la victoire de François Hollande, la presse française et étrangère a salué la fin du "président bling-bling» (voir par ex. El Périodico de Catalunya : «El fin del bling-bling ») en l’opposant au "président normal» (voir La Voz de Galicia : "Fin de “monsieur bling-bling”, triunfo de “monsieur normal” »). Par ailleurs, le Daily mail titre le même jour en français «Au revoir Président bling bling". 


\section{Références}

AUthIER-ReVUz Jacqueline, 1994, «L'énonciateur glosateur de ses mots. Explication et interprétation », Langue française, $\mathrm{n}^{0} 103$, p. 91-102.

BOYER Henri, 1990, “"Piche”, mot-stigmate et marqueur d'identité », L'identité, textes réunis par François Pic, Montpellier, Section française de l'Association internationale d'études occitanes, p. 21-33.

- 2003, De l'autre côté du discours, Paris, L'Harmattan.

Charaudeau Patrick, 1983, Langage et discours. Éléments de sémiolinguistique (Théorie et pratique), Paris, Hachette.

- 1992, Grammaire du sens et de l'expression, Paris, Hachette éducation.

Dakhlia Jamil, Lherault Marie, 2008, "Peopolisation et politique», Le Temps des Médias, nº10.

GALISSON Robert, 1988, "Cultures et lexicultures. Pour une approche dictionnairique de la culture partagée», Annexes des Cahiers de linguistique hispanique médiévale, vol. VII, Hommage à Bernard Pottier, p. 325-341.

Guilbert Louis, 1973, "Théorie du néologisme», Cahiers de l'Association internationale des études françaises, n² 25, p. 9-29.

LAURENDEAu Paul, 1997, «De la "déformabilité" des notions en discours ", Langage et société, n०82, p. 27-47.

LOCHARD Guy, BOYER Henri, 1998, La communication médiatique, Paris, Le Seuil.

PRUVOST Jean, 2005, «Quelques concepts lexicographiques opératoires à promouvoir au seuil du xxıe siècle», Ela. Études de linguistique appliquée, 2005/1, nº137, p.7-37.

Pruvost Jean, SABLAYrolles Jean-François, 2003, Les néologismes, Paris, PUF (Que sais-je?).

Reboul Olivier, 1980, Langage et idéologie, Paris, PUF.

SABLAYROLles Jean-François, 1993, "La double motivation de certains néologismes», Faits de langues, no 1 , p. 223-226.

Steuckardt Agnès, Honoré Jean-Paul éd., 2006, L'emprunt et sa glose, Mots. Les langages du politique, n82, Lyon, ENSÉditions.

WINDISCH Uli, 1987, Le K.-O. verbal. La communication conflictuelle, Giromagny, L’Âge d'Homme. 


\section{Annexe}

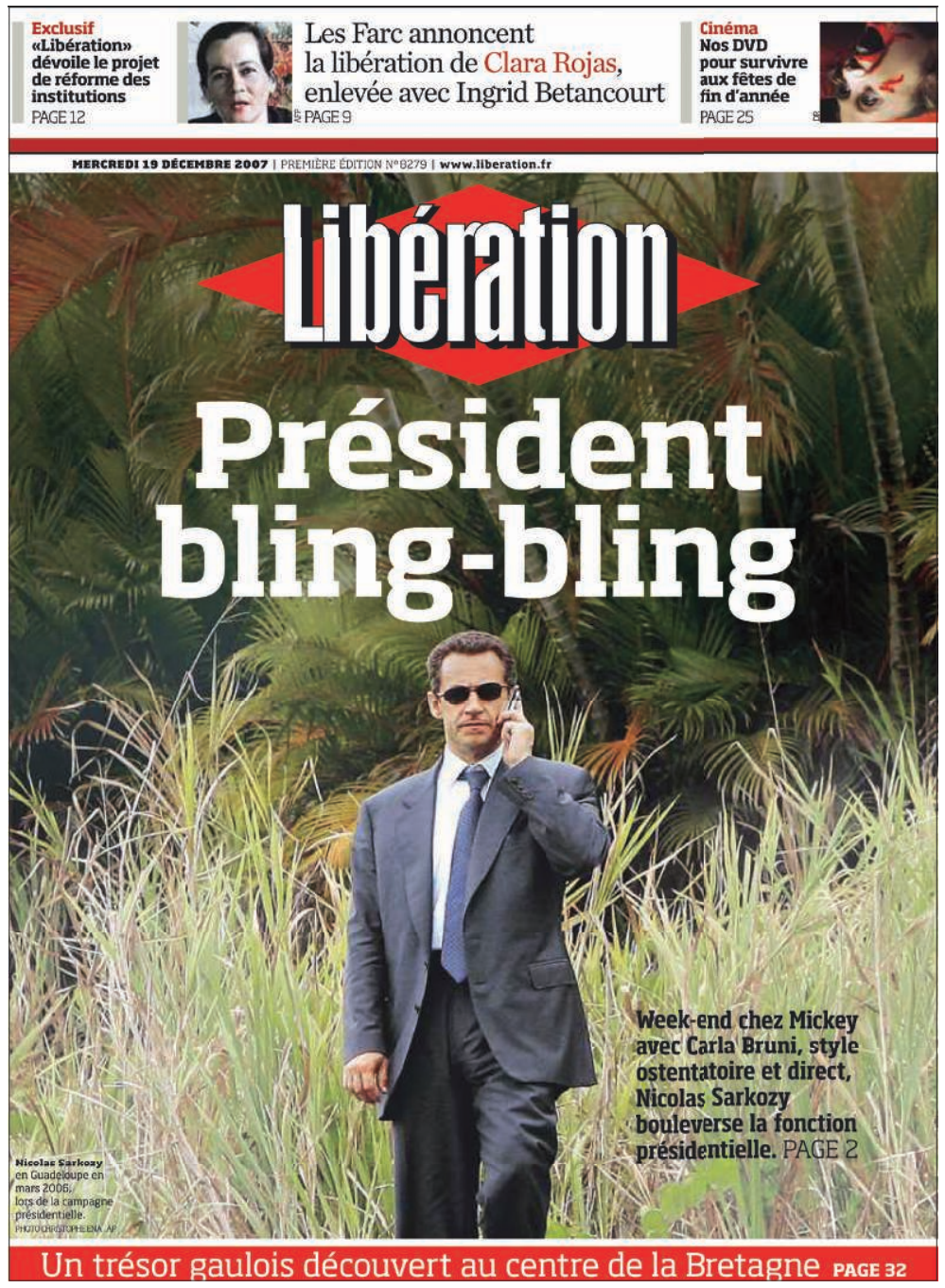

(C) Photo Christophe Ena - AP / Libération / 19122007 